\title{
Study on the properties of the two types of channel wave with $3 D$ finite-difference method
}

\author{
Yuge Jia ${ }^{1}$, Dechun $\mathrm{Li}^{3}$, Wenqiang Yang ${ }^{4}$ \\ School of Resource and Earth Science \\ China University of Mining and Technology \\ Xuzhou 221008, China \\ gegecumt@126.com
}

\author{
Jiulong Cheng ${ }^{2}$ \\ College of Geoscience and Surveying Engineering \\ China University of Mining and Technology \\ Beijing 100083, China
}

\begin{abstract}
This article studies the formation, propagation and spatial distribution of the two types of channel wave in three-dimensional space, using three-dimensional inhomogeneous isotropic medium wave equation and solving the elastic wave equation with finite-difference method. Geological model designed in this paper are symmetric model about coal seam. At final we analysis the formation, propagation characteristics and spatial distribution of the two types of channel wave, compare distinction and connection of the two types of channel wave and then provide a theoretical basis and guidance for the channel wave exploration.
\end{abstract}

Keywords - Love-channel wave;Rayleigh-channelwave; finite-difference method;numerical simulation

\section{INTRODUCTION}

Coal mining geological environment is very complex. During the process of coal mining,geological anomaly body (i.e. coal seam discontinuity, such as gangue, small faults, collapse column, variation in thickness of coal seam, etc.) that has not been provenin front of the working face will result in mining process and technology more complicated. Therefore, before and during mining process, it is very important to detect the small geological structure in front of the working face.

Using channel wave seismic exploration method, the fine geological structure can be effectively identified. It is carried outin coal seam mining working faceunderground. Source excitation and seismic wave received are along the coal tunnel layout. Channel wave is very sensitive to abnormal body and geological dataknown are the basis of the data interpretation. Therefore channel waveseismic exploration is the best underground seismic exploration method with most effective, most accurateand highest resolution.

\section{THE FORMATION AND CLASSIFICATION OF CHANNEL WAVE}

When the seismic wave is excited in the low velocity layer, the velocity of surrounding rock is relatively high. When the incident angle is greater than critical angle, it will totally reflect at the top and bottom interface. Totally reflection will reverberate and superimpose mutually with refraction. The major wave energy is limited in low velocity layer and not leak into surrounding rock. The low coal seam is like a waveguide layer and this phenomenon is called seismic wave guide phenomenon, also known as channel wave, as shown in Fig.1.

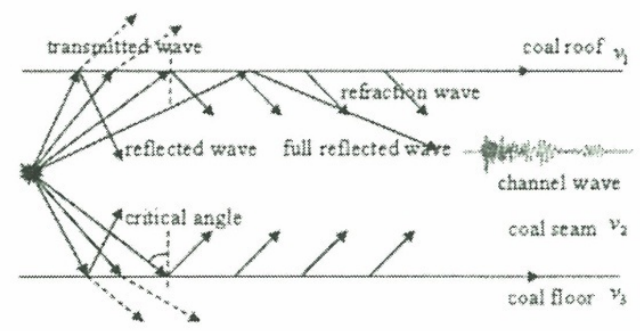

Fig 1. The formation of channel wave

\section{A. The Formation of the Channel Wave}

There are direct waves (direct $\mathrm{P}$ wave and $\mathrm{S}$ wave) and channel wave (Love-channel wave and Rayleigh-channel wave) in seismic wave record. Channel wave is formed by the superposition of various types of wave and the velocity of channel wave is the function of frequency. Because the channel wave is confined in the coal seam, the propagation distance is large in the coal seam and the channel wave is related to the thickness of the coal seam.

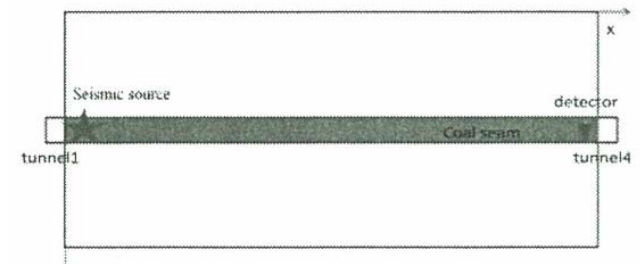

Fig.2 Profile of geological model

\section{B. The Classification of the Channel Wave}

When Love-channelwave propagate, the particles movein horizontalplaneand perpendicular to the direction of the direction of wave propagation, similar to the hunting motion.

When Rayleigh-channelwave propagate, the particlesmove in the vertical plane along the direction of the wave propagation and the movement is a reverse ellipse, i.e.moving forward, upward, backward and downward. 
Channel wave is the surface wave propagation in coal seamand the channel wave is also divided into two types, i.e.

\section{FINITE DIFFERENCE NUMERICAL SOLUTION OF WAVE}

\section{EQUATION}

\section{A. The inhomogeneous isotropic elastic wave equation}

satisfied byChannel wave

Eq. (1) is for the inhomogeneous elasticwave with the isotropic medium ${ }^{[1,2,3,4]}$.
Love-channel wave and Rayleigh-channel wave.

$$
\left\{\begin{array}{l}
\frac{\partial^{2} u_{x}}{\partial t^{2}}=\left(\frac{\lambda+2 \mu}{\rho}\right)\left[\frac{\partial^{2} u_{x}}{\partial x^{2}}+\frac{\partial^{2} u_{y}}{\partial x \partial y}+\frac{\partial^{2} u_{z}}{\partial x \partial z}\right]+\left(\frac{\mu}{\rho}\right)\left[\frac{\partial^{2} u_{x}}{\partial y^{2}}+\frac{\partial^{2} u_{x}}{\partial z^{2}}-\frac{\partial^{2} u_{y}}{\partial x \partial y}-\frac{\partial^{2} u_{z}}{\partial x \partial z}\right] \\
\frac{\partial^{2} u_{y}}{\partial t^{2}}=\left(\frac{\lambda+2 \mu}{\rho}\right)\left[\frac{\partial^{2} u_{x}}{\partial x \partial z}+\frac{\partial^{2} u_{y}}{\partial y^{2}}+\frac{\partial^{2} u_{z}}{\partial y \partial z}\right]+\left(\frac{\mu}{\rho}\right)\left[\frac{\partial^{2} u_{y}}{\partial x^{2}}+\frac{\partial^{2} u_{y}}{\partial z^{2}}-\frac{\partial^{2} u_{x}}{\partial x \partial y}-\frac{\partial^{2} u_{z}}{\partial y \partial z}\right] \\
\frac{\partial^{2} u_{x}}{\partial t^{2}}=\left(\frac{\lambda+2 \mu}{\rho}\right)\left[\frac{\partial^{2} u_{x}}{\partial x \partial z}+\frac{\partial^{2} u_{y}}{\partial y \partial z}+\frac{\partial^{2} u_{z}}{\partial z^{2}}\right]+\left(\frac{\mu}{\rho}\right)\left[\frac{\partial^{2} u_{z}}{\partial x^{2}}+\frac{\partial^{2} u_{z}}{\partial y^{2}}-\frac{\partial^{2} u_{x}}{\partial x \partial z}-\frac{\partial^{2} u_{y}}{\partial y \partial z}\right]
\end{array}\right.
$$

In witch, $\lambda$ and $\mu$ are Lame coefficient. $u_{x}, u_{y}$ and $u_{z}$ are components of displacement.

When centralized source is excited along the y-direction, Love-channelwaveoccupies the main wave along the X-direction. As shown in Fig.3 and Fig.4, the Y component of the wave mainly indicates Love-channelwave.

When the explosive source is excited, along $\mathrm{X}$-direction, $\mathrm{X}$ componentmainly indicates Rayleigh-channelwave. Along y-direction, $\quad \mathrm{Y}$ componentmainly indicates Rayleigh-channelwave, as shown in Fig.5 and Fig.6.

\section{Geological Model and Parameters}

In this paper, we solve the Equation (1) using finite-difference method. Profile of geological model is shown in Fig.2.

Uniform grid is $1 m \times 1 m \times 1 m$ and model scale is $M=200 \Delta x \times 100 \Delta y \times 100 \Delta z$. The main frequency of Ricker wavelet is $100 \mathrm{~Hz}$ and the time sampling interval is $\Delta t=0.0002 \mathrm{~s}$.

Seismic source locates at $x=10 \mathrm{~m}, y=50 \mathrm{~m}, z=50 \mathrm{~m}$. The thickness of coal seam is $4 \mathrm{~m}$.

The parameters in the geological model are shown in TABLE I.
TABLE I. Elastic Parameters of GeOlogical Model

\begin{tabular}{|l|c|c|c|}
\hline & $V_{p}(\mathrm{~m} / \mathrm{s})$ & $V_{s}(\mathrm{~m} / \mathrm{s})$ & $\rho\left(\mathrm{kg} / \mathrm{m}^{3}\right)$ \\
\hline 1 Roof & 2600 & 1800 & 2500 \\
\hline 2Coal seam & 2000 & 1200 & 1400 \\
\hline 3 Bottom & 2600 & 1800 & 2500 \\
\hline
\end{tabular}

\section{ThreE Dimensional WAVE Field SNAPSHOT}

In this paper we calculated the centralized source (mainly excite Love-channel wave) and the explosion source (mainly excite Rayleigh-channel wave). For each type of source we calculated two cross section, both $x-z$ and $x-y$.

Two types of channel wave at $t=0.1 s$ are shown in Fig.3, Fig. 4, Fig. 5 and Fig.6.

\section{A. Centralized Source (Mainly Excite Love-channel Wave)}

As shown in Fig. 3 and Fig. 4, the characteristics of love-channel wave field snapshots are as follows:

- Along the $\mathrm{x}$-direction, wave is mainly in love-channel wave, i.e. Y component.

- $\quad$ Rayleigh-channel wave is very weak, it can be seen that the vertical component of the Rayleigh wave is perpendicular to the coal seamand easily leaked into the surrounding rock.

- $\quad$ Comparing Fig.3(a) and (c), the vertical component $\mathrm{Z}$ is weaker 1 magnitude than the horizontal component $\mathrm{X}$. 
Rayleigh-channel wave is mainly in horizontal component.

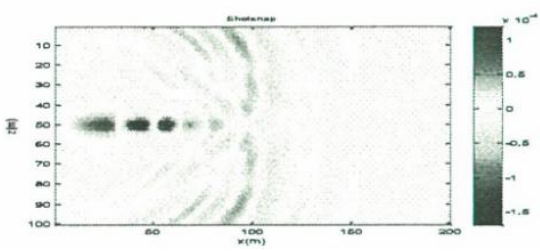

(a) X component

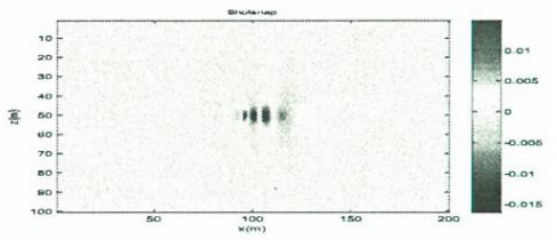

(b) Y component

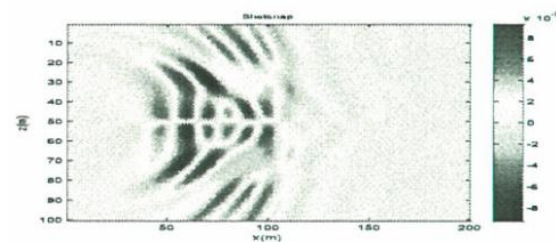

(c) $\mathrm{Z}$ component

Fig. 3. $\quad x-z$ wave field snapshot

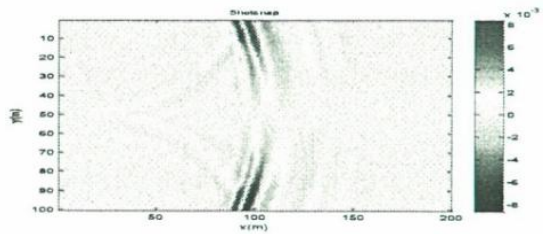

(a) X component

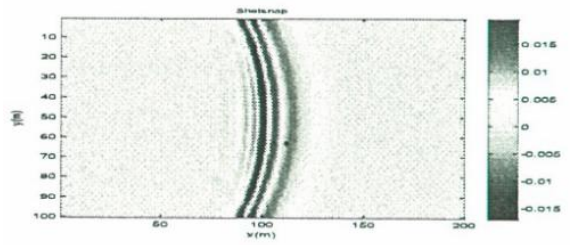

(b) Y component

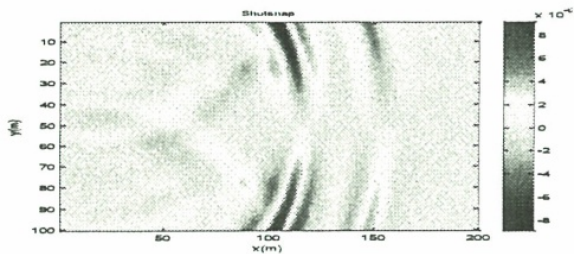

(c) Z component

Fig. 4. $x-y$ wave field snapshot

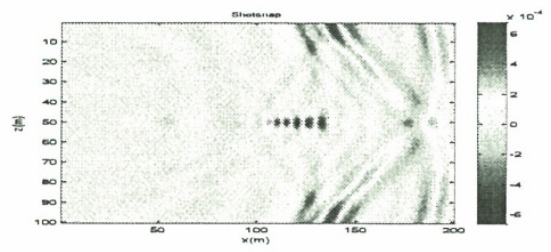

(a) X component

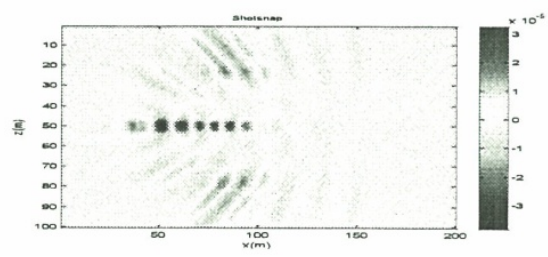

(b) Y component

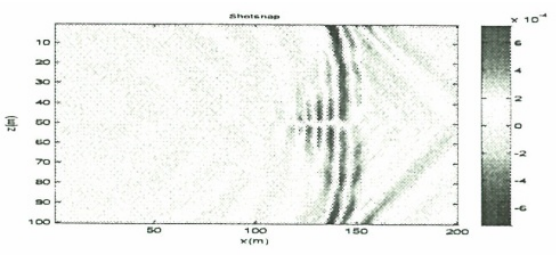

(c) $\mathrm{Z}$ component

Fig. 5. $x-z$ wave field snapshot

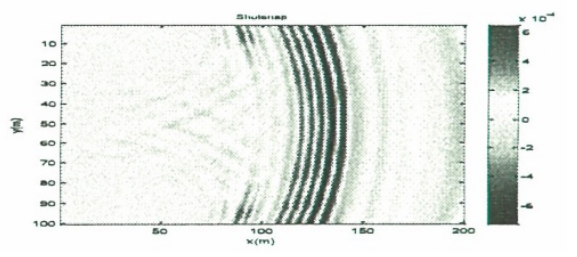

(a) X component

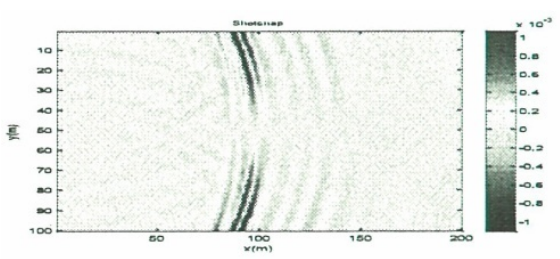

(b) Y component

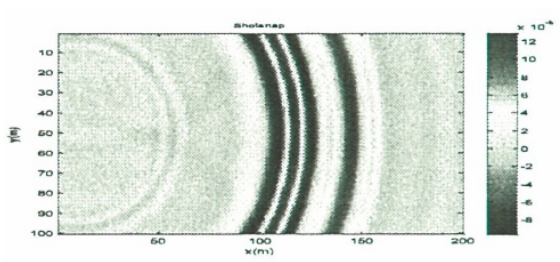

(c) $\mathrm{Z}$ component

Fig. 6. $x-y$ wave field snapshot 


\section{B. Explosion Source (Mainly Excite Rayleigh-channel Wave)}

As shown in Fig. 5 and Fig. 6, explosion source mainly excite Rayleigh-channel wave and the characteristics of wave field snapshots are as follows:

- Along the $\mathrm{x}$-direction, wave is mainly in Rayleigh-channel wave, i.e. $\mathrm{X}$ component.

- Along the y-direction, wave is mainly in Rayleigh-channel wave, i.e. Y component.

- It can be seen that the vertical component of the Rayleigh wave is perpendicular to the coal seamand easily leaked into the surrounding rock.

\section{CONCLUSIONS}

Through the comparative analysis of the calculation results, finally we get the following understanding:

- Love-channel wave exploration, in fact, is to excite and receive Love-channel wave. As shown in Fig. 3 and Fig. 4, Centralized source is excited intunnel 1 and two horizontal component of the channel wave are received in the tunnel 4 . The more the wave deviated from the $\mathrm{x}$-direction, the-Lovechannel wave is more weak.The transmission channel wave exploration is mainly based on the Love-channel wave exploration.

- Rayleigh-channel wave exploration, in fact, is to excite and receive Rayleigh-channel wave. As shown in Fig. 5and Fig. 6. The reflection channel wave exploration is mainly based on the Rayleigh-channel wave exploration.

- Whether it is the Love-channel wave explorationor the Rayleigh-channel waveexploration, the vertical component of the wave mainly leak into the surrounding rock and the vertical component of the received energy is weak. Therefore in practical work, channel waveseismic explorationmostly use two horizontal component records.

\section{ACKNOWLEDGMENTS}

This work was funded by the National Natural Science Fund (NNSF, Project: 41304112)

\section{REFERENCE}

[1] Yang S T, Cheng J L. Numerical simulation of fore detecting with seismic in coal roadway and study of wave field characteristics. Journal of China Coal Society(in Chinese)[J], 2010, 35(10): $1633 \sim 1637$.

[2] G Z, Cheng J Y, Zhu P M, et a1. 3-D numerical simulation and dispersion analysis of in-seam wave in underground coalmine[J]. Chinese J. Geophys. (in Chinese), 2012, 55(2): 645 654.

[3] Yang Z, Ge M C, Wang S G. Characteristics of transmittingchannel wave in a coal seam [J]. Mining Science \&Technology, 2009, 19: $331 \sim 336$.

[4] Zhu G M, Li G H, Cheng J Y. Numerical simulation ofseismic survey in coal mine roadway[J]. Journal of ChinaCoal Society[J], 2008, 33(11): $1263 \sim 1267$ 